\title{
BMJ Open Use of psychotropic drugs and drugs with anticholinergic properties among residents with dementia in intermediate care facilities for older adults in Japan: a cohort study
}

\author{
Shota Hamada (D) , ${ }^{1,2,3}$ Taro Kojima, ${ }^{4}$ Yukari Hattori, ${ }^{4}$ Hiroshi Maruoka, ${ }^{5}$ \\ Shinya Ishii, ${ }^{6}$ Jiro Okochi, ${ }^{3,7}$ Masahiro Akishita ${ }^{4}$
}

To cite: Hamada S, Kojima T, Hattori Y, et al. Use of psychotropic drugs and drugs with anticholinergic properties among residents with dementia in intermediate care facilities for older adults in Japan: a cohort study. BMJ Open 2021;11:e045787. doi:10.1136/ bmjopen-2020-045787

- Prepublication history and additional materials for this paper is available online. To view these files, please visit the journal online (http://dx.doi. org/10.1136/bmjopen-2020045787).

Received 13 October 2020 Revised 27 February 2021 Accepted 22 March 2021

Check for updates

(C) Author(s) (or their employer(s)) 2021. Re-use permitted under CC BY-NC. No commercial re-use. See rights and permissions. Published by BMJ.

For numbered affiliations see end of article.

Correspondence to

Dr Shota Hamada;

shota.hamada@ihep.jp

\section{ABSTRACT}

Objectives To evaluate the prescription and

discontinuation of psychotropic drugs (PD) and drugs with anticholinergic properties (DAP) in residents with dementia admitted to Roken, a major type of long-term care facility in Japan.

Design Cohort study.

Setting A nationwide questionnaire survey across 3598

Roken in Japan in 2015 (up to five randomly selected residents per facility).

Participants This study included 1201 residents from 343 Roken (response rate: $10 \%$ ). We determined the presence and severity of dementia using a nationally standardised measure.

\section{Primary and secondary outcome}

measures Prescriptions of PD and DAP at admission and 2 months after admission were evaluated. Multivariable logistic regression was used to evaluate the associations of residents' baseline characteristics with prescriptions or discontinuation.

Results Prescription rates decreased for antidementia drugs $(19.4 \%$ to $13.0 \%)$, hypnotics $(25.1 \%$ to $22.6 \%)$ and anxiolytics (12.3\% to $10.7 \%)$, whereas those for other PD, such as antipsychotics (13.2\% to $13.6 \%)$, antidepressants $(7.4 \%$ to $6.7 \%)$, antiepileptic drugs $(7.1 \%$ to $7.8 \%)$ and DAP $(35.2 \%$ to $36.6 \%)$ did not statistically significantly decrease. Some factors were associated with the prescriptions, for example, for antipsychotics, older age ( $\geq 85$ years) (adjusted $\mathrm{OR}(\mathrm{aOR}), 0.60 ; 95 \% \mathrm{Cl} 0.43$ to 0.85 ) and being bedridden (aOR $0.67 ; 95 \% \mathrm{Cl} 0.47$ to 0.97 ) were associated with a lower use of antipsychotics, whereas severe dementia was associated with a higher use of antipsychotics (aOR 3.26; 95\% Cl 2.26 to 4.70). At an individual level, a quarter of residents prescribed $\mathrm{PD}$ or DAP at admission had discontinued at least one PD or DAP respectively, 2 months after admission. Antidementia drug use in severe dementia (aOR 1.86; 95\% $\mathrm{Cl} 1.04$ to 3.31) and PD use in older age (aOR 1.61; 95\% $\mathrm{Cl} 1.00$ to 2.60; in residents with disabling dementia) were associated with discontinuation

Conclusions There is possible scope for deprescribing PD and DAP in Roken residents with dementia to mitigate the risks of adverse events.
Strengths and limitations of this study

- This analysis was undertaken using the largest available dataset on drug use in Roken facilities.

- The presence and severity of dementia were determined using a nationally standardised measure.

- The study sample analysed cannot be considered representative of Roken residents nationwide because the survey response rate on medication was only approximately $10 \%$.

- Data were not analysed according to facility-level or medical director-level because too few residents were studied per facility.

- The generalisability of our findings may be limited to similar types of nursing homes or long-term care facilities only.

\section{INTRODUCTION}

There are approximately 50 million people with dementia worldwide, and this number is projected to triple in the next 30 years. $^{1}$ Dementia and cognitive impairment are wellevidenced predictors of nursing home $(\mathrm{NH})$ placement, ${ }^{2}$ and the prevalence of dementia is considerably higher in $\mathrm{NH}$ residents compared with community-dwelling older adults. ${ }^{3}$ Most NH or long-term care facility (NH/LTCF) residents have dementia. ${ }^{3-5}$ People with dementia manifest not only memory impairments but also typically exhibit a variety of behavioural and psychological symptoms of dementia (BPSD), including aggression, agitation, depression, psychosis and sleep problems. ${ }^{6}{ }^{7}$ The complexity involved in managing BPSD remains a major challenge in dementia care.

Residents of NH/LTCFs are commonly prescribed a large number of drugs (ie, polypharmacy) including potentially inappropriate medications (PIM) for older adults, ${ }^{89}$ 
and optimisation of medication use is often required for better clinical outcomes. Although non-pharmacological approaches should first be considered for the management of BPSD,${ }^{10}$ pharmacological treatments, such as antipsychotics, benzodiazepines and antidepressants, have been commonly administered to NH/LTCF residents despite safety concerns and limited effectiveness. ${ }^{11-19}$ Furthermore, wide variations in the use of these types of drugs have been reported among different countries. ${ }^{20}$

In terms of safety concerns related to medication for BPSD management, the following issues have been reported. Antipsychotic use in individuals with dementia was associated with greater risks of cerebrovascular events and mortality. ${ }^{21}$ The use of psychotropic drugs (PD), such as sedatives/hypnotics, antidepressants and benzodiazepines, was associated with an increased risk of falls. ${ }^{22}$ Moreover, an increased number of PD prescribed was associated with the greater risks of fall injuries, hospitalisations and mortality. ${ }^{23}$ Drugs with anticholinergic properties (DAP) were associated with greater risks for cognitive impairment, falls and mortality. ${ }^{24}$ Given that NH/LTCF residents are frail and more susceptible to adverse events compared with community-dwelling older people, careful pharmacological management approaches should be undertaken.

Intermediate care facilities for older adults with longterm care (LTC) needs (Geriatric Health Service Facilities or Roken) are a major type of LTCFs in Japan. The main role of these facilities is providing medical and LTC services, including pharmacotherapy and rehabilitation, to improve residents' functioning and to facilitate their return home. We previously reported that the prescription of antidementia drugs decreased after admission, whereas antipsychotic prescription remained stable after admission to Roken ${ }^{25}$; however, dementia severity had not been considered. We also reported that residents with severe dementia were less likely to be prescribed drugs to prevent and treat cardiovascular diseases, such as antihypertensives and lipid-modifying drugs ${ }^{26}$ However, data on the use of PD and DAP in Roken residents are scarce. Therefore, we aimed to evaluate the utilisation of PD and DAP in Roken residents with dementia in Japan. We also aimed to identify factors associated with prescription and discontinuation of PD and DAP among Roken residents.

\section{METHODS}

\section{Setting}

Roken is one of three types of LTCFs in Japan covered by public LTC insurance systems, and $>90 \%$ of Roken are private sector operated. ${ }^{27}$ According to a national survey conducted in 2015, namely, the 'Survey of Institutions and Establishments for long-term care,' the total capacity of Roken facilities was 368201 residents nationwide. ${ }^{28}$ This figure corresponded to approximately $1 \%$ of older people aged $\geq 65$ years or $8 \%$ of older people with LTC needs. The majority of older adults are admitted to a facility after discharge from a hospital. ${ }^{27}$ Roken residents are stable in terms of medical conditions, but they require specific care and rehabilitation prior to returning home. The mean length of stay has been reported to be approximately 10 months. ${ }^{27}$ General services in Roken comprise routine medical and LTC services, with some exceptions.

Fees for medical, including pharmacotherapy and rehabilitation, and LTC services provided in Roken are paid to the facility as a bundled payment from public LTC insurance systems. The fees have been determined based on LTC needs for individual residents, the facility subtype of Roken, and on additional services, but not on drug costs. Out-of-pocket payment rates for Roken services generally comprise $10 \%$ of total expenditures. Therefore, in most cases, changes to medication do not affect residents' outof-pocket payments.

In general, a full-time medical director in a facility is in charge of and responsible for medication, and pharmacists, nurses and other staff contribute information to support decisions concerning residents' medication. Information regarding medication prescribed at admission is generally obtained from a drug information sheet issued by a physician in charge of an individual prior to admission to a facility. Social workers and other facility staff may obtain additional information from staff members in a hospital prior to admission or from primary care professionals.

\section{Data source}

We sent questionnaires via mail to obtain data regarding drug utilisation to 3598 Roken in 2015. Details on the survey have been previously reported. ${ }^{25}$ This data collection method was applied at that time because medical claims data for drugs were not generally issued for Roken residents and most Roken facilities had not introduced standardised electronic systems to record drug prescriptions.

Briefly, we collected data for up to five randomly selected individuals per Roken who were admitted between August and October 2015. The survey was conducted after approval from the ethics committee of the Japan Association of Geriatric Health Services Facilities (No. 30 in 2015 and No. 37 in 2016). All participants or their representatives provided written informed consent, and all data were collected after anonymisation at each facility. Medical directors or facility managers in individual Roken facilities were responsible for responding to the survey. No residents or their families were involved in completing the survey.

\section{Definition of dementia}

We previously reported that about half of the residents had dementia in this dataset, based on reported diagnoses of any types of dementia and/or prescription of antidementia drugs. ${ }^{25}$ However, because the diagnosis of dementia had not been always reported and because only a subset of residents with dementia had been treated with antidementia drugs, ${ }^{4} 29$ we identified residents with dementia in the present study using a nationally 
standardised measure to assess cognitive function ('Independence Degree of Daily Living for the Dementia'). ${ }^{30}$ This unique measure in Japan has been verified to identify dementia and has relatively high sensitivity and specificity. ${ }^{31}$ Therefore, we expected to identify residents with dementia while avoiding the inclusion of residents without dementia. More specifically, we selected residents with dementia if they had rank I (having dementia but almost independent for daily living) or higher based on this measure; this cut-off was previously reported to have a sensitivity of $65 \%$ and a specificity of $93 \% .{ }^{31}$ Dementia severity was categorised into two groups for this study: mild-to-moderate (rank I and rank II (having daily life-disturbing symptoms, behaviours and problems in communication but independent for daily life if watched by someone) ) and severe (rank III (requiring assistance), rank IV (requiring assistance frequently) and rank M (marked psychiatric symptoms requiring expert management)). A recent study also used this measure to identify older adults with dementia and to estimate the prevalence of antipsychotic use. ${ }^{32}$

\section{Study participants}

The selection of study participants has been previously described. ${ }^{25}{ }^{26}$ From data collected using the survey on 1510 residents at 368 Roken (response rate: 10.2\%), we first selected residents aged $\geq 65$ years who had stayed at the same facility for at least two consecutive months after admission. Residents with missing demographic data and those with no record of any drugs prescribed both at admission and 2 months after admission (possible missing data for drug prescriptions) were excluded. Accordingly, data from 1324 participants at 350 facilities were obtained after removing data due to ambiguous drug names, strengths and dosages. We selected 1318 residents with records of physical and cognitive functions assessed using nationally standardised measures. ${ }^{30}$ Finally, among them, we selected 1201 residents $(91.1 \%)$ with dementia (as defined above) from 343 Roken facilities for further analyses.

\section{Drugs of interest}

In the survey, we asked to collect data on regularly scheduled medications only; however, as-needed drugs were occasionally reported. In this study, we omitted the data on as-needed drugs due to inconsistent reporting from facilities. Therefore, we evaluated the utilisation of regular drugs administered at admission and at 2 months after admission.

Four marketed antidementia drugs were examined, namely, three acetylcholinesterase inhibitors (donepezil, galantamine and rivastigmine) and one N-methylD-aspartate receptor antagonist (memantine). PD comprised the following six categories: antipsychotics (first generation and second generation), antidepressants, hypnotics, anxiolytics, antiepileptic drugs and lithium (online supplemental table S1). In addition, the use of yokukansan, a traditional Japanese Kampo medicine, was also studied although this drug was not included in the PD categories. A meta-analysis of randomised controlled trials showed that yokukansan had beneficial effects on some symptoms related to dementia, including delusions, hallucinations and agitation/aggression. ${ }^{33}$

Various scales to identify and assess anticholinergic burdens have been developed and validated, but with relatively low concordance. ${ }^{34}$ We identified drugs in 11 DAP categories, which were available in Japan, for screening purposes according to drugs listed in the 'Guidance of Appropriate Medication for Elderly Patients' released in May 2018 in Japan, ${ }^{35}$ the Anticholinergic Risk Scale ${ }^{36}$ and/or the Beers criteria (online supplemental table S2). ${ }^{37}$

\section{Statistical analysis}

Descriptive statistics were used to summarise the characteristics of the study participants and prescriptions of drugs. Physical status was categorised as non-bedridden (ranks J and $\mathrm{A}$ ) and bedridden (ranks $\mathrm{B}$ and $\mathrm{C}$ ) ${ }^{30}$ The bedridden group included residents who lay in bed most of the day but were able to sit (rank B).$^{30}$ The characteristics of residents according to dementia severity were compared using a $\chi^{2}$ test, and a Wilcoxon rank sum test was also used for age. Changes in prescriptions from admission to 2 months after admission were tested using the McNemar test. We defined discontinuation as no prescription for antidementia drugs or individual categories of PD as well as a decrease in the number of PD or DAP at 2 months after admission among users of the corresponding drugs at admission. Multivariable logistic regression analysis was used to evaluate the associations between residents' baseline characteristics and prescription or discontinuation of antidementia drugs, PD and DAP, and relevant adjusted odds ratios (aOR) and 95\% CI were reported. Independent variables included age group (65-84 and $\geq 85$ years), sex, dementia severity (mild-to-moderate and severe) and physical disability (non-bedridden and bedridden). As a sensitivity analysis, we repeated the above analyses in residents with 'disabling' dementia (ranks II or higher). ${ }^{30} 31$ All analyses were conducted using Stata V.14 (StataCorp).

\section{Patient and public involvement}

No patients or members of the public were involved in the study design, conduct, reporting, or dissemination plans in relation to this research.

\section{RESULTS}

\section{Baseline characteristics}

Study participants' baseline characteristics are shown in table 1 and in online supplemental table S3. This study included 1201 residents with dementia, of whom $47 \%$ exhibited severe dementia, $75 \%$ were women and $60 \%$ were $\geq 85$ years old. Residents with severe dementia were more likely to have a physical disability (ie, be bedridden) compared with those with mild-to-moderate dementia $(\mathrm{p}<0.001)$. 
Table 1 Characteristics of residents with dementia at admission according to dementia severity

\begin{tabular}{|c|c|c|c|c|}
\hline & \multirow[b]{2}{*}{$\begin{array}{l}\text { Overall } \\
(n=1201)\end{array}$} & \multicolumn{2}{|c|}{ Dementia severity } & \multirow[b]{2}{*}{$P$ value } \\
\hline & & $\begin{array}{l}\text { Mild-to- } \\
\text { moderate } \\
(\mathrm{n}=642)\end{array}$ & $\begin{array}{l}\text { Severe } \\
(n=559)\end{array}$ & \\
\hline \multicolumn{5}{|l|}{ Age (years) } \\
\hline $65-74$ & $97(8.1)$ & $54(8.4)$ & $43(7.7)$ & 0.815 \\
\hline $75-84$ & $381(32.7)$ & 209 (32.6) & $172(30.8)$ & \\
\hline $85-94$ & $612(51.0)$ & $319(49.7)$ & $293(52.4)$ & \\
\hline $95+$ & $111(9.2)$ & $60(9.3)$ & $51(9.1)$ & \\
\hline Mean \pm SD & $86 \pm 7$ & $85 \pm 7$ & $86 \pm 7$ & 0.483 \\
\hline \multicolumn{5}{|l|}{ Sex } \\
\hline Men & $298(24.8)$ & $150(23.4)$ & $148(26.5)$ & 0.213 \\
\hline Women & 903 (75.2) & $492(76.6)$ & 411 (73.5) & \\
\hline \multicolumn{5}{|l|}{ Physical disability } \\
\hline Non-bedridden & $398(33.1)$ & $274(42.7)$ & $124(22.2)$ & $<0.001$ \\
\hline Bedridden & 803 (66.9) & 368 (57.3) & 435 (77.8) & \\
\hline
\end{tabular}

$\mathrm{SD}$, standard deviation

\section{Drug utilisation}

The utilisation of antidementia drugs, $\mathrm{PD}$ and DAP is shown in table 2 and in online supplemental table S4. The use of antidementia drugs decreased from admission to 2 months after admission in all participants and according to dementia severity $($ all $\mathrm{p}<0.001)$. The relative decrease was more pronounced in residents with severe dementia (23.8\% to $14.3 \%$; $39.9 \%$ decrease) than in those with mild-to-moderate dementia ( $15.6 \%$ to $11.8 \%$; $24.4 \%$ decrease).

At least one category of PD was prescribed to $>40 \%$ of the residents. The most frequently prescribed drug category was hypnotics, followed by antipsychotics, anxiolytics, antidepressants and antiepileptic drugs. Among residents with antiepileptic drugs, $23(27.1 \%)$ residents at admission and $24(25.5 \%)$ residents at 2 months after admission had a recorded diagnosis of symptomatic epilepsy. Lithium was rarely prescribed. The prescription rates for hypnotics and anxiolytics decreased from admission to 2 months after admission. The use of yokukansan did not change from admission $(n=63,5.2 \%)$ to 2 months after admission $(\mathrm{n}=56,4.7 \%)$ (exact $\mathrm{p}=0.248)$.

When benzodiazepines and Z-drugs, categorised as hypnotics or anxiolytics, were evaluated together, the proportion of residents receiving these kinds of drugs decreased from $29.8 \%(\mathrm{n}=358)$ at admission to $27.8 \%$ $(\mathrm{n}=334)$ at 2 months after admission $(\mathrm{p}=0.012)$ in all participants. The overall decrease observed in all participants was attributed to a decrease in prescriptions for residents with mild-to-moderate dementia $(\mathrm{n}=213(33.2 \%)$ to $\mathrm{n}=193(30.1 \%), \mathrm{p}=0.003)$, in contrast to those with severe

Table 2 Utilisation of antidementia drugs, psychotropic drugs and drugs with anticholinergic properties

\begin{tabular}{|c|c|c|c|c|}
\hline & $\begin{array}{l}\text { Admission } \\
\mathrm{n}(\%)\end{array}$ & $\begin{array}{l}\text { Month } 2 \\
\mathrm{n}(\%)\end{array}$ & $P$ value & $\begin{array}{l}\text { Discontinuation } \\
\mathrm{n} \text { (\% of users at admission) }\end{array}$ \\
\hline Antidementia drugs & 233 (19.4) & 156 (13.0) & $<0.001$ & $84(36.1)$ \\
\hline Psychotropic drugs & $532(44.3)$ & $514(42.8)$ & 0.089 & $113(23.2)^{\star}$ \\
\hline Hypnotics & $301(25.1)$ & $272(22.6)$ & 0.002 & $58(19.3)$ \\
\hline Antipsychotics & $158(13.2)$ & $163(13.6)$ & 0.535 & $30(19.0)$ \\
\hline First generation & $68(5.7)$ & $70(5.8)$ & 0.715 & $14(20.6)$ \\
\hline Second generation & $101(8.4)$ & $105(8.7)$ & 0.564 & $22(21.8)$ \\
\hline Anxiolytics & $148(12.3)$ & $128(10.7)$ & 0.003 & $33(22.3)$ \\
\hline Antidepressants & $89(7.4)$ & $81(6.7)$ & $0.134 \dagger$ & $15(16.9)$ \\
\hline Antiepileptic drugs & $85(7.1)$ & $94(7.8)$ & $0.049 \dagger$ & $4(4.7)$ \\
\hline Lithium & $2(0.2)$ & $2(0.2)$ & NA & $0(0.0)$ \\
\hline Drugs with anticholinergic properties & $423(35.2)$ & $440(36.6)$ & 0.141 & $77(26.1)^{\star}$ \\
\hline \multicolumn{5}{|l|}{ Frequently prescribed drugs $\ddagger$} \\
\hline H2-receptor antagonists & $162(13.5)$ & $191(15.9)$ & $<0.001$ & $20(12.3)$ \\
\hline Antipsychotics & $106(8.8)$ & $113(9.4)$ & 0.336 & $23(21.7)$ \\
\hline Drugs for overactive bladder & $73(6.1)$ & $69(5.7)$ & $0.541 \dagger$ & 14 (19.2) \\
\hline Drugs for Parkinson's disease & $56(4.7)$ & $54(4.5)$ & $0.774 \dagger$ & 7 (12.5) \\
\hline Antidepressants & $55(4.6)$ & $55(4.6)$ & $1.000 \dagger$ & $8(14.5)$ \\
\hline
\end{tabular}

*Discontinuation of psychotropic drugs (PD) or drugs with anticholinergic properties (DAP) overall was defined as a decrease in the number of drug categories from PD or DAP, from admission to month 2, respectively.

†Exact $p$ values are shown in cases where a small number of residents (less than 30 residents) changed the prescription.

$\ddagger$ See online supplemental table S2 for specific drugs included in each drug category.

NA, not available. 
dementia where no such decrease was observed ( $\mathrm{n}=145$ $(25.9 \%)$ to $\mathrm{n}=141(25.2 \%), \mathrm{p}=0.564)$.

More than one-third of study participants were prescribed DAP, and this proportion did not change from admission to 2 months after admission (for all participants, $35.2 \%$ to $36.6 \%, p=0.165)$. H2-blockers were the most frequently prescribed DAP, and the prescription rate increased from admission to 2 months after admission in all participants $(13.5 \%$ to $15.9 \%, \mathrm{p}<0.001)$. Famotidine accounted for $80 \%$ of H2-blockers at admission and 2 months after admission. Related to the increased use of H2-blockers, the prescription of proton pump inhibitors decreased from $32.2 \% \quad(n=387)$ at admission to $26.3 \%$ $(\mathrm{n}=316)$ at 2 months after admission. Antipsychotics and muscarinic receptor antagonists for overactive bladder were also frequently prescribed DAP.

Regarding changes in the use of drugs at an individual level, more than one-third of residents who were prescribed antidementia drugs at admission and $>20 \%$ of residents who were prescribed PD or DAP at admission were discontinued the corresponding drugs at 2 months after admission. Only seven residents started antidementia drugs after admission, but the number of residents who started antipsychotics $(n=35)$ or DAP $(n=94)$ was comparable to those who discontinued $(\mathrm{n}=30$ or 113 , respectively). The number of residents who started hypnotics, anxiolytics, or antidepressants was about half the number of residents who discontinued.

\section{Factors associated with prescriptions}

The associations between residents' baseline characteristics and the prescriptions of antidementia drugs, PD and DAP are shown in table 3 . Older age ( $\geq 85$ years) was associated with fewer prescriptions of antipsychotics, hypnotics, anxiolytics, antidepressants, antiepileptic drugs and DAP. Women were more likely to receive antidementia drugs and tended to receive anxiolytics more frequently. Residents with severe dementia were more likely to be prescribed antidementia drugs, antipsychotics, antiepileptic drugs and DAP. In addition, they tended to receive anxiolytics less frequently. Bedridden residents were less likely to be prescribed antidementia drugs and antipsychotics and tended to receive hypnotics less frequently. Similar results were obtained from the sensitivity analysis in residents with disabling dementia (online supplemental table S5).

\section{Factors associated with discontinuation}

Discontinuation of antidementia drugs was more likely in residents with severe dementia (aOR, 1.86; 95\% CI 1.04 to $3.31 ; \mathrm{p}=0.035$ ) (table 4 ). In residents with disabling dementia, those aged $\geq 85$ years were more likely to have discontinued the use of PD (aOR, 1.61; 95\% CI 1.00 to 2.60; $\mathrm{p}=0.049$ ) (online supplemental table $\mathrm{S} 6$ ).

\section{DISCUSSION}

In this study, we examined the utilisation of antidementia drugs, PD and DAP in Roken residents and identified

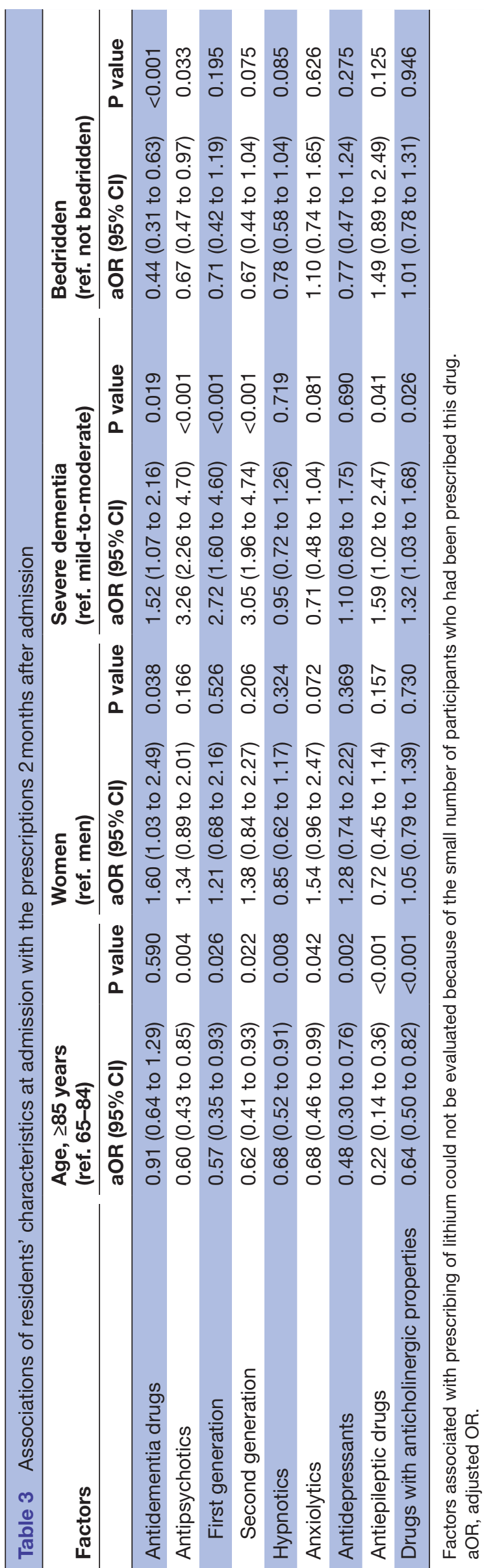




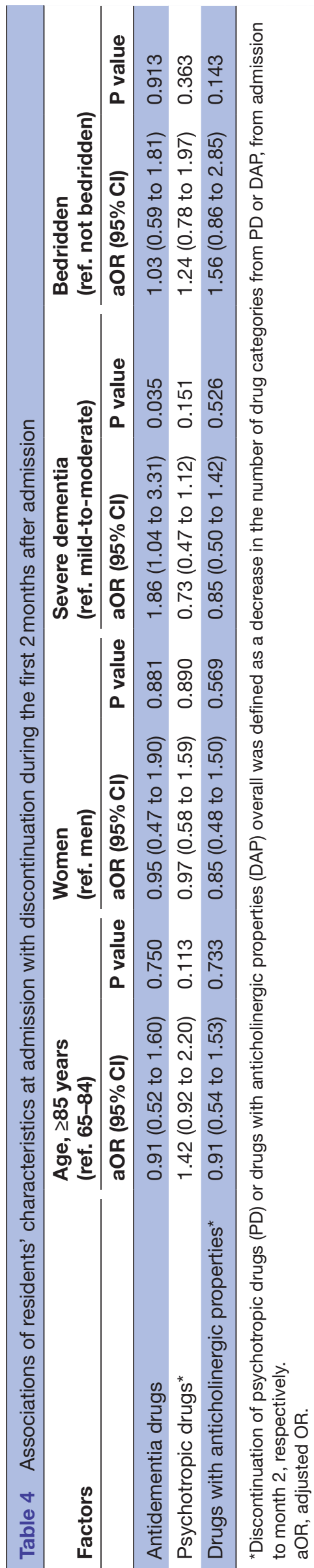

factors associated with prescription or discontinuation of these drugs. These findings have further characterised drug utilisation in Roken residents in addition to our findings from previous studies. ${ }^{252638}{ }^{39}$ We previously reported that residents with severe dementia received a smaller number of drugs and were less frequently prescribed antihypertensives or lipid-modifying drugs compared with those with better cognitive function. ${ }^{26}$ Despite our observations concerning the discontinuation of PD and DAP, some drugs, especially antipsychotics and DAP, were commonly initiated. Antidementia drugs for severe dementia and PD for older aged residents (with disabling dementia) were identified as being associated with discontinuation. Larger studies are needed to evaluate discontinuation of individual drug categories in relation to PD or DAP.

Antidementia drugs were discontinued in about onethird of residents receiving these drugs at admission, and residents with severe dementia were more likely to have had their antidementia drugs discontinued. This may be explained as due to the expected limited effectiveness of antidementia drugs, especially for those with severe dementia, or due to intolerability or issues concerning adverse effects. The proportion of residents who received antidementia drugs was higher in our study, despite the decrease after admission, than in studies involving $\mathrm{NH}$ residents with dementia or those not limited to dementia that have been conducted in other countries. ${ }^{12} 1617$ We did not obtain any information on non-pharmacological approaches in this dataset, but some residents who discontinued antidementia drugs may have received rehabilitation for dementia. ${ }^{40}$ Another plausible reason for discontinuation of antidementia drugs might have been to reduce the financial burden incurred by a facility due to the bundled payment system, including drug costs, applying to Roken. ${ }^{25}$

Compared with a study conducted in some different types of NH/LTCFs in Japan, the prevalence of antipsychotics was slightly lower in our study sample, whereas the prevalence of antidepressants and hypnotics/anxiolytics was comparable. ${ }^{18}$ We observed a decreased use of hypnotics and anxiolytics in residents with mild-tomoderate dementia, which led to a decrease in regularly prescribed PD after admission to Roken. The decrease may result from adjustment of the use of hypnotics and anxiolytics in response to symptom changes. ${ }^{18}$ In contrast, there were no changes in the prescription of PD in residents with severe dementia found in our study. Therefore, our results suggest that challenges concerning the management of BPSD in residents with severe dementia still remain.

Several studies have been conducted to evaluate the utilisation of PD and DAP in NH/LTCF residents with and without dementia in other countries. Studies conducted in Europe and Australia have reported that half to threequarters of NH/LTCF residents received at least one $\mathrm{PD}$, which was more frequent than in our study. ${ }^{11} 14161720$ The prevalence of antipsychotics varied between studies, 
ranging from about 10\%-60\% of residents. ${ }^{11} 1214161720$ The relatively low prescription rate of antipsychotics in Roken residents is encouraging, especially because of the lower likelihood of their being prescribed to the older population as well as to those with reduced physical function. However, greater efforts to avoid the use of antipsychotics may be possible in Roken, with improved staffing, resources and staff education. ${ }^{641}$ Some successful measures to reduce antipsychotic prescriptions in NHs have been reported in other countries, such as the USA and Canada ${ }^{42}{ }^{43}$; however, similar regulatory initiatives have not yet been implemented in Japan.

Hypnotics were found to be the most frequently prescribed PD in the present study, with a decrease in prescription after admission to a facility. In total, a quarter of Roken residents were prescribed hypnotics, which was comparable to findings in a study involving $\mathrm{NH} /$ LTCF residents in Norway (22.9\% in 2009), ${ }^{14}$ but nearly double the prevalence of hypnotics reported in a study in Germany and in Austria (9.9\% to 13.3\%). ${ }^{11}$ In Japan, the frequent use of hypnotics is not limited to NH/LTCF residents, as it has also been reported in the outpatient setting. ${ }^{445}$ Taken together, the frequent use of hypnotics in Roken residents suggests the need for continuous monitoring of the appropriate use of hypnotics in NH/ LTCFs and other community or hospital settings.

The prevalence of anxiolytics in our study was similar to that found in studies from European countries. It was reported that the prevalence of anxiolytics in NH residents was $22.2 \%$ in Austria and $10.9 \%$ and $12.9 \%$ in two different cohorts in Germany, and men were less likely to use anxiolytics. ${ }^{11}$ Furthermore, a study from Norway observed an increase in the use of anxiolytics from $14.9 \%$ in 1997 to $21.9 \%$ in 2009 , and reported that women and younger residents (age, $<80$ years) were more likely to use anxiolytics. ${ }^{14}$ Therefore, the associations of sex and age with the prescription of anxiolytics observed in our study are consistent with those previous findings. ${ }^{11} 14$

About $7 \%$ of Roken residents with dementia were prescribed antidepressants, and no apparent changes in prescription were observed during 2 months of observation. The prevalence of antidepressants was twice as high as that in older outpatients aged $\geq 75$ years $(3.7 \%)$ in Japan. ${ }^{45}$ However, the prescription rate in our study was considerably lower compared with that reported in studies conducted in other countries, with approximately one-fifth to two-thirds of $\mathrm{NH}$ residents with or without dementia reported to have received antidepressants. ${ }^{11-1720}$ We also found that older age was associated with infrequent use of antidepressants, which is consistent with findings from previous studies; however, we did not observe a significantly greater use of antidepressants in women, which has been reported in previous studies. ${ }^{1114}$ The lower prevalence of antidepressants may be attributed to the preferred use of anxiolytic benzodiazepines instead of antidepressants for residents with mild depression or anxiety. ${ }^{13} 46$ Future studies are needed to verify the appropriateness of prescribing antidepressants, including the possibility of underuse, in relation to diagnosis and severity of depression and other indications..$^{13}$

We conducted screening for DAP with reference to lists provided in Japan's national guidelines document ('Guidance of Appropriate Medication for Elderly Patients'), the Anticholinergic Risk Scale and Beers criteria, without quantification of anticholinergic burdens. We found that more than one-third of Roken residents with dementia received DAP. In a study from Helsinki, half of the residents of NHs or assisted living facilities received DAP based on the Anticholinergic Risk Scale. ${ }^{19}$ In our study, frequently prescribed DAP included H2-blockers, antipsychotics, and muscarinic receptor antagonists for overactive bladder. Some proton pump inhibitors were replaced with H2-blockers, possibly due to safety concerns, such as kidney diseases, infections, and fractures. ${ }^{47}$ Anticholinergic burden due to H2-blockers should also be considered, especially in the case of concomitant use of other DAP. The prevalence of muscarinic receptor antagonists for overactive bladder was similar to that found in a nationwide study of Roken residents $(5.7 \%) .{ }^{48}$ Given that two-thirds of Roken residents had urinary incontinence, ${ }^{48}$ the prescription rate might be too low. A study involving $\mathrm{NH}$ residents in the USA indicated the possibility of an unmet need for treating urinary incontinence in $\mathrm{NH}$ / LTCF residents. ${ }^{49}$

This study has several limitations. First, the appropriateness of the prescribed medications could not be determined because we did not collect data regarding the indications for each drug or the duration of treatment. However, most of the drugs studied were PIM, and thus have been considered inappropriate for this population. ${ }^{50}$ Similarly, we did not determine the processes or reasons for the discontinuation of drugs, and further studies are needed to evaluate the appropriateness of drug discontinuation. Second, we did not analyse the data according to facility-level or medical director-level because too few residents were studied per facility. Therefore, there may be variations in prescriptions among different facilities, as previously observed in antipsychotic prescription rates reported in the USA. ${ }^{51}$ Third, we could not ensure the representativeness of the present study sample in terms of residents of Roken nationwide because the survey response rate on medications was only approximately $10 \%$. However, the current dataset analysed was the largest available on drug use in Roken facilities. Fourth, our study findings are dependent on the accuracy of the data collected and the items covered. Directions for as-needed drug use by medical directors might not always have been reported. We considered that these limitations may cause overestimation of the prevalence of drugs because of drugs without directions possibly being included as regularly scheduled drugs, or underestimation in cases involving as-needed drugs, which may have been important components of usual medications. As-needed drugs should also be considered as part of complex drug regimens for NH/LTCF residents in future studies. ${ }^{52}$ Fifth, we identified associations 
between residents' characteristics and prescriptions, but only limited variables were considered; therefore, there is a possibility of residual confounding, such as in relation to the types of dementia involved. Finally, the generalisability of our findings may be limited to similar types of $\mathrm{NH} /$ LTCFs only.

In conclusion, $\mathrm{PD}$ and DAP were commonly prescribed for Roken residents with dementia. Taken together, there appears to be scope for deprescription of PD and DAP in Roken residents with dementia to mitigate the risks of adverse events, through engaging in careful reviews, including reviewing current indications and conducting assessments of adverse effects, because the risks in prescribing these drugs may outweigh the benefits.

\section{Author affiliations}

${ }^{1}$ Research Department, Institute for Health Economics and Policy, Association for Health Economics Research and Social Insurance and Welfare, Tokyo, Japan ${ }^{2}$ Department of Health Services Research, Faculty of Medicine, University of Tsukuba, Tsukuba, Japan

${ }^{3}$ Department of Home Care Medicine, Graduate School of Medicine, The University of Tokyo, Tokyo, Japan

${ }^{4}$ Department of Geriatric Medicine, Graduate School of Medicine, The University of Tokyo, Tokyo, Japan

${ }^{5}$ Yokohama Aobanosato Geriatric Health Services Facility, Yokohama, Japan ${ }^{6}$ Department of Medicine for Integrated Approach to Social Inclusion, Graduate School of Biomedical and Health Sciences, Hiroshima University, Hiroshima, Japan

${ }^{7}$ Tatsumanosato Geriatric Health Services Facility, Daito, Japan

Contributors SH, TK, SI, JO and MA contributed to the conception and design of the study. TK, $\mathrm{SI}, \mathrm{JO}$ and MA collected the data. SH conducted the statistical analysis, interpreted the results and drafted the manuscript. TK, YH, HM, SI, J0 and MA contributed to the interpretations of the results and revised the manuscript critically for important intellectual content. All authors approved the final manuscript.

Funding This study was supported by the Ministry of Health, Labour and Welfare, Japan (Grant Number H30-ninchisho-ippan-001 and research projects on health and welfare promotion for the elderly: №. 85 in FY2015 and No. 69 in FY2016).

Disclaimer The funding body had no involvement in study design, data collection, analysis and interpretation of data, writing of the report, or in the decision to submit the article for publication.

Competing interests $\mathrm{HM}$ and $\mathrm{JO}$ work at Roken. $\mathrm{JO}$ is an executive committee member of the Japan Association of Geriatric Health Services Facilities. MA received remuneration from Astellas Pharma, Boehringer Ingelheim, Daiichi Sankyo, Eisai, Eli Lilly Japan, Mitsubishi-Tanabe Pharma, Mochida Pharmaceutical, MSD, Ono Pharmaceutical, Pfizer Japan, Sumitomo Dainippon Pharma, and Takeda Pharmaceutical, and research funding from Astellas Pharma, AstraZeneca, Bayer Health Care, Boehringer Ingelheim, Chugai Pharmaceutical, Daiichi Sankyo, Eli Lilly Japan, Kowa Pharmaceutical, Mitsubishi-Tanabe Pharma, Mochida Pharmaceutical, MSD, Ono Pharmaceutical, Otsuka Pharmaceutical, Pfizer Japan, Taisho Toyama Pharmaceutical, Takeda Pharmaceutical, and Tsumura. The other authors declare no conflicts of interest regarding this study.

Patient consent for publication Not required.

Provenance and peer review Not commissioned; externally peer reviewed.

Data availability statement Data may be obtained from a third party and are not publicly available. Data that support our study's findings are available from the corresponding author on reasonable request after permission from the Japan Association of Geriatric Health Services Facilities.

Supplemental material This content has been supplied by the author(s). It has not been vetted by BMJ Publishing Group Limited (BMJ) and may not have been peer-reviewed. Any opinions or recommendations discussed are solely those of the author(s) and are not endorsed by BMJ. BMJ disclaims all liability and responsibility arising from any reliance placed on the content. Where the content includes any translated material, BMJ does not warrant the accuracy and reliability of the translations (including but not limited to local regulations, clinical guidelines, terminology, drug names and drug dosages), and is not responsible for any error and/or omissions arising from translation and adaptation or otherwise.

Open access This is an open access article distributed in accordance with the Creative Commons Attribution Non Commercial (CC BY-NC 4.0) license, which permits others to distribute, remix, adapt, build upon this work non-commercially, and license their derivative works on different terms, provided the original work is properly cited, appropriate credit is given, any changes made indicated, and the use is non-commercial. See: http://creativecommons.org/licenses/by-nc/4.0/.

ORCID iD

Shota Hamada http://orcid.org/0000-0003-4939-1834

\section{REFERENCES}

1 World Health Organization. Dementia. Available: https://www.who.int/ news-room/fact-sheets/detail/dementia [Accessed 4 Sep 2020].

2 Luppa M, Luck T, Weyerer S, et al. Prediction of institutionalization in the elderly. A systematic review. Age Ageing 2010;39:31-8.

3 Hoffmann F, Kaduszkiewicz H, Glaeske G, et al. Prevalence of dementia in nursing home and community-dwelling older adults in Germany. Aging Clin Exp Res 2014;26:555-9.

4 Lithgow S, Jackson GA, Browne D. Estimating the prevalence of dementia: cognitive screening in Glasgow nursing homes. Int $J$ Geriatr Psychiatry 2012;27:785-91.

5 Stewart R, Hotopf M, Dewey M, et al. Current prevalence of dementia, depression and behavioural problems in the older adult care home sector: the South East London care home survey. Age Ageing 2014;43:562-7.

6 Kales HC, Gitlin LN, Lyketsos CG. Assessment and management of behavioral and psychological symptoms of dementia. BMJ 2015;350:h369.

7 Zhao Q-F, Tan L, Wang H-F, et al. The prevalence of neuropsychiatric symptoms in Alzheimer's disease: Systematic review and metaanalysis. J Affect Disord 2016;190:264-71.

8 Jokanovic N, Tan ECK, Dooley MJ, et al. Prevalence and factors associated with polypharmacy in long-term care facilities: a systematic review. J Am Med Dir Assoc 2015;16:535.e1-e12.

9 Morin L, Laroche M-L, Texier G, et al. Prevalence of potentially inappropriate medication use in older adults living in nursing homes: a systematic review. J Am Med Dir Assoc 2016;17:862.e1-e9.

10 Azermai M, Petrovic M, Elseviers MM, et al. Systematic appraisal of dementia guidelines for the management of behavioural and psychological symptoms. Ageing Res Rev 2012;11:78-86.

11 Richter T, Mann E, Meyer G, et al. Prevalence of psychotropic medication use among German and Austrian nursing home residents: a comparison of 3 cohorts. J Am Med Dir Assoc 2012;13:187. e7-e13.

12 Onder G, Liperoti R, Fialova D, et al. Polypharmacy in nursing home in Europe: results from the shelter study. J Gerontol A Biol Sci Med Sci 2012;67:698-704.

13 Bourgeois J, Elseviers MM, Van Bortel L. The use of antidepressants in Belgian nursing homes: focus on indications and dosages in the PHEBE study. Drugs Aging 2012;29:759-69.

14 Ruths S, Sørensen PH, Kirkevold Øyvind, et al. Trends in psychotropic drug prescribing in Norwegian nursing homes from 1997 to 2009: a comparison of six cohorts. Int J Geriatr Psychiatry 2013;28:868-76.

15 Midlöv P, Andersson M, Östgren CJ, et al. Depression and use of antidepressants in Swedish nursing homes: a 12-month follow-up study. Int Psychogeriatr 2014;26:669-75.

16 Nyborg G, Brekke M, Straand J, et al. Potentially inappropriate medication use in nursing homes: an observational study using the NORGEP-NH criteria. BMC Geriatr 2017;17:220.

17 McMaster M, Fielding E, Lim D, et al. A cross-sectional examination of the prevalence of psychotropic medications for people living with dementia in Australian long-term care facilities: issues of concern. Int Psychogeriatr 2018;30:1019-26.

18 Ozaki T, Katsumata Y, Arai A. Association between changes in the use of psychotropic drugs and behavioural and psychological symptoms of dementia over 1 year among long-term care facility residents. Psychogeriatrics 2019;19:126-34.

19 Aalto UL, Roitto H-M, Finne-Soveri H, et al. Temporal trends in the use of anticholinergic drugs among older people living in long-term care facilities in Helsinki. Drugs Aging 2020;37:27-34.

20 Janus SIM, van Manen JG, IJzerman MJ, et al. Psychotropic drug prescriptions in Western European nursing homes. Int Psychogeriatr 2016;28:1775-90. 
21 Tampi RR, Tampi DJ, Balachandran S, et al. Antipsychotic use in dementia: a systematic review of benefits and risks from metaanalyses. Ther Adv Chronic Dis 2016;7:229-45.

22 Woolcott JCet al. Meta-analysis of the impact of 9 medication classes on falls in elderly persons. Arch Intern Med 2009;169:1952-60.

23 Johnell K, Jonasdottir Bergman G, Fastbom J, et al. Psychotropic drugs and the risk of fall injuries, hospitalisations and mortality among older adults. Int J Geriatr Psychiatry 2017;32:414-20.

24 Ruxton K, Woodman RJ, Mangoni AA. Drugs with anticholinergic effects and cognitive impairment, falls and all-cause mortality in older adults: a systematic review and meta-analysis. $\mathrm{Br} J \mathrm{Clin}$ Pharmacol 2015;80:209-20.

25 Hamada S, Kojima T, Sakata N, et al. Drug costs in long-term care facilities under a per diem bundled payment scheme in Japan. Geriatr Gerontol Int 2019;19:667-72.

26 Hamada S, Kojima T, Maruoka H, et al. Utilization of drugs for the management of cardiovascular diseases at intermediate care facilities for older adults in Japan. Arch Gerontol Geriatr 2020;88:104016.

27 Ministry of Health, Labour and Welfare. Survey of institutions and establishments for long-term care, 2016.

28 Ministry of Health, Labour and Welfare. Survey of institutions and establishments for long-term care, 2015.

29 Dyer SM, Gnanamanickam ES, Liu E, et al. Diagnosis of dementia in residential aged care settings in Australia: an opportunity for improvements in quality of care? Australas J Ageing 2018;37:E155-8.

30 Kawagoe S, Tsuda T, Doi H. Study on the factors determining home death of patients during home care: a historical cohort study at a home care support clinic. Geriatr Gerontol Int 2013;13:874-80.

31 Noda H, Yamagishi K, Ikeda A, et al. Identification of dementia using standard clinical assessments by primary care physicians in Japan. Geriatr Gerontol Int 2018;18:738-44.

32 Kuroda N, Hamada S, Sakata N, et al. Antipsychotic use and related factors among people with dementia aged 75 years or older in $\mathrm{J}$ apan: A comprehensive population-based estimation using medical and long-term care data. Int J Geriatr Psychiatry 2019;34:472-9.

33 Matsunaga S, Kishi T, Iwata N. Yokukansan in the treatment of behavioral and psychological symptoms of dementia: an updated meta-analysis of randomized controlled trials. JAD 2016;54:635-43.

34 Naples JG, Marcum ZA, Perera S, et al. Concordance between anticholinergic burden scales. J Am Geriatr Soc 2015;63:2120-4.

35 Ministry of Health, Labour, and Welfare. Guidance of appropriate medication for elderly patients (General), 2018.

36 Rudolph JLet al. The anticholinergic risk scale and anticholinergic adverse effects in older persons. Arch Intern Med 2008;168:508-13.

37 By the American Geriatrics Society 2015 Beers Criteria Update Expert Panel. American geriatrics Society 2015 updated beers criteria for potentially inappropriate medication use in older adults. $J$ Am Geriatr Soc 2015;63:2227-46.
38 Hamada S, Ohno Y, Kojima T, et al. Prevalence of cytochrome P450-mediated potential drug-drug interactions in residents of intermediate care facilities for older adults in Japan. Geriatr Gerontol Int 2019:19:513-7.

39 Hamada S, Kojima T, Sakata N, et al. Changes in use of high-priced new drugs and drug costs among residents of long-term care facilities under a bundled payment system: case studies of dipeptidyl peptidase-4 inhibitors and direct oral anticoagulants. Geriatr Gerontol Int 2020;20:842-4.

40 Maki Y, Sakurai T, Okochi J, et al. Rehabilitation to live better with dementia. Geriatr Gerontol Int 2018;18:1529-36.

41 Cousins JM, Bereznicki LRE, Cooling NB, et al. Prescribing of psychotropic medication for nursing home residents with dementia: a general practitioner survey. Clin Interv Aging 2017;12:1573-8.

42 Bowblis JR, Lucas JA, Brunt CS. The effects of antipsychotic quality reporting on antipsychotic and psychoactive medication use. Health Serv Res 2015;50:1069-87.

43 Ivers NM, Taljaard M, Giannakeas V, et al. Public reporting of antipsychotic prescribing in nursing homes: population-based interrupted time series analyses. BMJ Qual Saf 2019;28:121-31.

44 Suzuki Y, Sakakibara M, Shiraishi N, et al. Prescription of potentially inappropriate medications to older adults. A nationwide survey at dispensing pharmacies in Japan. Arch Gerontol Geriatr 2018;77:8-12.

45 Ishizaki T, Mitsutake S, Hamada S, et al. Drug prescription patterns and factors associated with polypharmacy in $>1$ million older adults in Tokyo. Geriatr Gerontol Int 2020;20:304-11.

46 Nakagawa A, Williams A, Sado M. Comparison of treatment selections by Japanese and US psychiatrists for major depressive disorder: a case vignette study. Psychiatry Clin Neurosci 2015;69:553-62.

47 Schoenfeld AJ, Grady D. Adverse effects associated with proton pump inhibitors. JAMA Intern Med 2016;176:172-4.

48 Suzuki M, Okochi J, lijima K, et al. Nationwide survey of continence status among older adult residents living in long-term care facilities in Japan: the prevalence and associated risk factors of incontinence and effect of comprehensive care on continence status. Geriatr Gerontol Int 2020;20:285-90.

49 Narayanan S, Cerulli A, Kahler K, et al. Is drug therapy for urinary incontinence used optimally in long-term care facilities? J Am Med Dir Assoc 2007;8:98-104.

50 Kojima T, Mizukami K, Tomita N, et al. Screening Tool for Older Persons' Appropriate Prescriptions for Japanese: Report of the Japan Geriatrics Society Working Group on "Guidelines for medical treatment and its safety in the elderly". Geriatr Gerontol Int 2016;16:983-1001

51 Chen Yet al. Unexplained variation across US nursing homes in antipsychotic prescribing rates. Arch Intern Med 2010;170:89-95.

52 Dörks M, Allers K, Hoffmann F. Pro re NatA drug use in nursing home residents: a systematic review. J Am Med Dir Assoc 2019;20:287-93. 